\title{
Patterns of care and outcomes of patients with METAstatic soft tissue SARComa in a real-life setting: the METASARC observational study
}

Marion Savina ${ }^{1,2}$, Axel Le Cesne ${ }^{3}$, Jean-Yves Blay ${ }^{4}$, Isabelle Ray-Coquard ${ }^{4}$, Olivier Mirr ${ }^{3}$, Maud Toulmonde ${ }^{5}$, Sophie Cousin ${ }^{5}$, Philippe Terrier ${ }^{6}$, Dominique Ranchere-Vince ${ }^{7}$, Pierre Meeus ${ }^{8}$, Eberhard Stoeckle ${ }^{9}$, Charles Honoré $^{10}$, Paul Sargos ${ }^{11}$, Marie-Pierre Sunyach ${ }^{12}$, Cécile Le Péchoux ${ }^{13}$, Antoine Giraud ${ }^{1}$, Carine Bellera ${ }^{1,2}$, François Le Loarer ${ }^{14}$ and Antoine Italiano ${ }^{5,15^{*}}$

\begin{abstract}
Background: Well-designed observational studies of individuals with rare tumors are needed to improve patient care, clinical investigations, and the education of healthcare professionals.

Methods: The patterns of care, outcomes, and prognostic factors of a cohort of 2225 patients with metastatic soft tissue sarcomas who were diagnosed between 1990 and 2013 and documented in the prospectively maintained database of the French Sarcoma Group were analyzed.

Results: The median number of systemic treatments was 3 (range, 1-6); 27\% of the patients did not receive any systemic treatment and 1054 (49\%) patients underwent locoregional treatment of the metastasis. Half of the patients who underwent chemotherapy $(n=810)$ received an off-label drug. Leiomyosarcoma was associated with a significantly better outcome than the other histological subtypes. With the exception of leiomyosarcomas, the benefit of a greater than third-line regimen was very limited, with a median time to next treatment (TNT) and overall survival (OS) ranging between 2.3 and 3.7 months and 5.4 and 8.5 months, respectively. The TNT was highly correlated with OS. Female sex, leiomyosarcoma histology, locoregional treatment of metastases, inclusion in a clinical trial, and treatment with first-line polychemotherapy were significantly associated with improved OS in the multivariate analysis.

Conclusions: The combination of doxorubicin with a second drug, such as ifosfamide, represents a valid option, particularly when tumor shrinkage is expected to provide clinical benefits. After failure of the second-line therapy, best supportive care should be considered, particularly in patients with non-leiomyosarcoma histology who are not eligible to participate in a clinical trial. Locoregional treatment of metastasis should always be included in the therapeutic strategy when feasible. TNT may represent a useful surrogate endpoint for OS in clinical studies.
\end{abstract}

Keywords: Sarcoma, Metastases, Outcome, Patterns of care, Chemotherapy, Surgery

\footnotetext{
* Correspondence: a.italiano@bordeaux.unicancer.fr

${ }^{5}$ Department of Medicine, Institut Bergonié, Bordeaux, France

${ }^{15}$ Early Phase Trials and Sarcoma Units, Institut Bergonié, 229 Cours de

l'Argonne, Bordeaux, France

Full list of author information is available at the end of the article
} 


\section{Background}

Soft-tissue sarcomas (STSs) represent a heterogeneous group of diseases that account for $1 \%$ of all malignancies in adults [1]. Despite adequate locoregional treatment, up to $40 \%$ of patients with STSs will develop metastatic disease $[1,2]$. When metastases are detected, the standard of care is based on palliative chemotherapy. Due to their rarity, no specific data on the comprehensive management and outcomes of metastatic STS patients are available.

A national network of care coordinated by three national reference centres has been set up through the support of the French National Cancer Institute for the management of STS patients. All suspected or diagnosed STS cases are reviewed by an accredited pathologist who is an expert in the field, and the cases are included in a national database. The aim of this study was to use this unique set of data to assess the modalities of treatment of patients with metastatic STS in a real-life setting, to evaluate their impact on the outcome according to the histological subtype, and to identify prognostic factors.

\section{Methods}

Patients

From 1990 to 2013, patients $\geq 18$ years old with a diagnosis of metastatic STS (excluding gastrointestinal stromal tumors, visceral sarcomas, and Ewing tumors) who were evaluated at one of the three national reference centres designated by the French National Cancer Institute for the management of STS (Centre Léon Bérard, Lyon; Institut Bergonié, Bordeaux; and Institut Gustave Roussy, Villejuif) were included in the prospectively maintained database of the French Sarcoma Group. A histological review of all patients was performed by the members of the pathological sub-committee of the French Sarcoma Group. The histological diagnosis and grading was established according to the World Health Organization Classification of Tumours and to the French grading system $[2,3]$.

\section{Outcomes}

Time to next treatment (TNT) was defined as the time from the systemic treatment onset to the next treatment or death due to any cause, whichever came first. When neither death nor new systemic therapy was observed, TNT was censored at the date of last patient contact. Overall survival (OS) was defined as the interval between the diagnosis of metastatic disease or the first-line systemic therapy onset and the time of death. When death was not observed, OS was censored at the date of last patient contact.

\section{Statistical analysis}

The statistical analysis of the baseline demographics and clinical outcomes was based on all data available up to the cut-off date of December 31, 2015. Descriptive statistics were used to show the distribution of variables in the population. Multivariate logistic regression models were used to identify biological and clinical factors associated with the type of treatment received and with the probability of survival 5 years after the diagnosis of metastases. Follow-up times were described as median values based on the inverse Kaplan-Meier estimator [4].

Prognostic factors of TNT and OS were identified using Cox proportional hazard models. The variables included in the univariate and multivariate analyses are detailed in Additional file 1.

The correlation between TNT and OS was evaluated at each of the four first-lines of metastatic chemotherapy by a Spearman rank correlation coefficient and was expressed as a value between 0 (no association) and 1 (perfect association). We used a reviewed copula-based approach that introduced an iterative multiple imputation method [5] for the estimation of the correlation coefficient. The data were analyzed using the SAS v9.3 and R v3.3 software packages.

\section{Results}

Patients

A total of 2165 patients were included in this study. Their characteristics are presented in Table 1 . The median follow-up duration was 61 months (range, 1-300). The five most frequently detected histological subtypes were leiomyosarcoma (LMS), undifferentiated pleomorphic sarcoma (UPS), synovial sarcoma (SS), dedifferentiated liposarcoma (DLPS), and malignant peripheral nerve sheath tumors (MPNST).

\section{General treatment patterns}

The general treatment patterns are described in Table 2. Patients over 75 years of age $(P<0.0001)$ and with MPNST $(P=0.0136)$ had a lower probability of receiving any systemic treatment, whereas presence of liver, lung, peritoneal, bone, pleural, skin, or lymphatic metastases was associated with a higher probability of receiving chemotherapy. Being over 75 years $(P<0.0001)$, DLPS $(P=0.0031)$, a grade $3(P$ $=0.0188)$, and the presence of more than one metastatic site $(P<0.0001)$ were associated with a lower probability of receiving a locoregional treatment, whereas being a woman $(P=0.0012)$, SS $(P=0.0026)$, and the presence of lymphatic, brain, bone, skin, soft tissue, or peritoneal metastases were associated with an increased probability of locoregional treatment. Locoregional metastasis treatment was the sole treatment for 250 patients (11.55\%). The metastasis localization was the only factor associated with the probability of receiving only locoregional treatment. Indeed, the presence of liver $(P<0.0001)$, lung $(P<$ 
Table 1 Patient characteristics according to the study population

\begin{tabular}{|c|c|c|c|c|c|c|}
\hline & \multirow{2}{*}{\multicolumn{2}{|c|}{$\frac{\text { All patients }}{(n=2165)}$}} & \multirow{2}{*}{\multicolumn{2}{|c|}{$\frac{\text { Patients alive at } 5 \text { years }}{(n=224)}$}} & \multirow{2}{*}{\multicolumn{2}{|c|}{$\begin{array}{l}\text { Patients treated with metastatic chemotherapy } \\
(\mathrm{n}=1575)\end{array}$}} \\
\hline & & & & & & \\
\hline & $n$ & $\%$ & $\mathrm{n}$ & $\%$ & $\mathrm{n}$ & $\%$ \\
\hline \multicolumn{7}{|l|}{ Sex } \\
\hline Male & 1055 & 48.73 & 92 & 41.07 & 754 & 47.87 \\
\hline Female & 1110 & 51.27 & 132 & 58.93 & 821 & 52.13 \\
\hline \multicolumn{7}{|l|}{ Age at first metastasis } \\
\hline$<75$ years old & 1886 & 87.11 & 216 & 96.43 & 1429 & 90.73 \\
\hline$\geq 75$ years old & 279 & 12.89 & 8 & 3.57 & 146 & 9.27 \\
\hline \multicolumn{7}{|l|}{ Histology } \\
\hline Leiomyosarcoma & 502 & 23.19 & 60 & 26.79 & 396 & 25.14 \\
\hline UPS & 203 & 9.38 & 9 & 4.02 & 141 & 8.95 \\
\hline DLPS & 172 & 7.94 & 12 & 5.36 & 112 & 7.11 \\
\hline Synovial sarcoma & 188 & 8.68 & 16 & 7.14 & 150 & 9.52 \\
\hline MPNST & 80 & 3.70 & 11 & 4.91 & 50 & 3.17 \\
\hline Other & 1020 & 47.11 & 116 & 51.79 & 726 & 46.10 \\
\hline \multicolumn{7}{|l|}{ Grade } \\
\hline 1 & 138 & 6.37 & 48 & 21.43 & 94 & 5.97 \\
\hline 2 & 590 & 27.25 & 74 & 33.04 & 440 & 27.94 \\
\hline 3 & 1083 & 50.02 & 63 & 28.13 & 765 & 48.57 \\
\hline Not available & 354 & 16.35 & 39 & 17.41 & 276 & 17.52 \\
\hline \multicolumn{7}{|c|}{ Number of metastatic sites } \\
\hline 1 & 1780 & 82.22 & 199 & 88.84 & 1248 & 79.24 \\
\hline$>1$ & 385 & 17.78 & 25 & 11.16 & 327 & 20.76 \\
\hline \multicolumn{7}{|l|}{ Metastatic sites } \\
\hline Lung & 1399 & 64.62 & 149 & 66.52 & 1075 & 68.25 \\
\hline Liver & 410 & 18.94 & 34 & 15.18 & 352 & 22.35 \\
\hline Peritoneum & 396 & 18.29 & 60 & 26.79 & 319 & 20.25 \\
\hline Bone & 370 & 17.09 & 29 & 12.95 & 305 & 19.37 \\
\hline Lymph node & 304 & 14.04 & 35 & 15.63 & 236 & 14.98 \\
\hline Skin & 172 & 7.94 & 25 & 11.16 & 136 & 8.63 \\
\hline Soft tissue & 173 & 7.99 & 36 & 16.07 & 135 & 8.57 \\
\hline Pleura & 163 & 7.53 & 11 & 4.91 & 140 & 8.89 \\
\hline Brain & 113 & 5.22 & 5 & 2.23 & 89 & 5.65 \\
\hline Bone marrow & 12 & 0.55 & 0 & 0.00 & 10 & 0.63 \\
\hline Other & 228 & 10.53 & 32 & 14.29 & 166 & 10.54 \\
\hline
\end{tabular}

UPS undifferentiated pleomorphic sarcoma, DLPS dedifferentiated liposarcoma, MPNST malignant peripheral nerve sheath tumors

0.0001), pleural $(P=0.0005)$, and peritoneal $(P=$ 0.0087 ) metastases was associated with a lower probability of locoregional treatment alone, whereas patients with soft-tissue metastases $(P=0.0031)$ were more likely to receive only a locoregional treatment. Best supportive care alone was more likely to be proposed to patients over 75 years $(P<0.0001)$, with a grade 3 tumor $(P=0.0306)$, or with multiple metastatic sites $(P=0.0201)$.

\section{Systemic treatment patterns (Table 2)}

The median number of systemic treatments received by the patients was $3(\min =1$ and $\max =6)$ and did not significantly differ across the histological subtypes. Patients $<75$ years old $(P<0.0001)$ and those with lymph node involvement $(P=0.0001)$ were more likely to receive polychemotherapy in the first-line setting. The most frequently prescribed off-label drug was gemcitabine. Female sex $(P=0.0313)$ and age $\geq 75$ years $(P=$ 
Table 2 General patterns of treatment according to study population

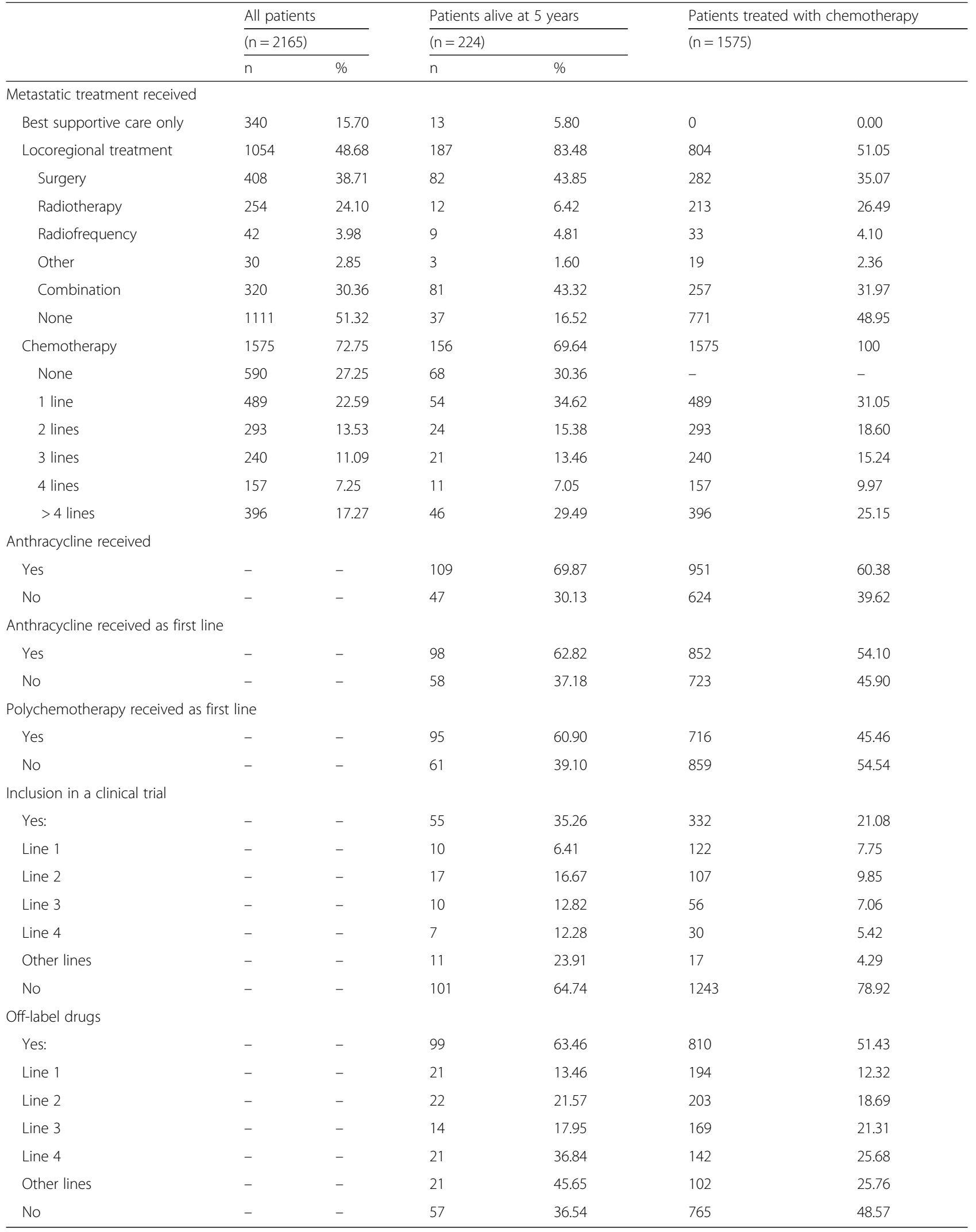


0.0003) were factors associated with a lower probability of being part of a clinical trial. On the contrary, patients with LMS or SS $(P=0.0217)$ and patients with liver $(P=$ $0.0072)$, skin $(P=0.0013)$ or peritoneal $(P=0.0036)$ metastases were more likely to be included in a clinical trial during the course of their treatment.

\section{Time to next treatment and overall survival}

The median TNT and OS according to the treatment line setting for the five most frequent histological subtypes are described in Table 3. Patients with metastatic LMS had the longest median survival, whereas patients with UPS had the shortest. The benefit of systemic therapy beyond the second line setting was limited, with a median TNT ranging between 2.3 and 3.5 months except for LMS (>4 months). The correlation estimated between TNT and OS was similar and high regardless of the considered chemotherapy line (rho $>0.65$ ); the highest value was observed in the first line setting $($ rho $=$ 0.76; 95\% CI, 0.73-0.78) (Table 4).

\section{Prognostic factors for time to next treatment}

We evaluated the prognostic TNT value calculated from the first line systemic therapy of the main biological, histological, and clinical factors for the 1575 patients who received at least one systemic treatment (Table 5).

Regarding the multivariate analysis, the following factors remained associated with an increased TNT: female sex, locoregional treatment of metastases, and administration of polychemotherapy in the first line of metastatic treatment (Table 5, Fig. 1). Only a grade 3 tumor at diagnosis remained associated with a decreased TNT (Table 5, Fig. 1).

\section{Prognostic factors for OS}

We evaluated the prognostic OS values of the main biological, histological, and clinical factors for the 1575

Table 3 Median time to next treatment (TNT) and overall survival (OS) according to the histological subtype and treatment setting

\begin{tabular}{lllll}
\hline & \multicolumn{4}{l}{ Median TNT/OS (months) } \\
\cline { 2 - 5 } & TNT1/OS1 $^{\mathrm{a}}$ & TNT2/OS2 $^{\mathrm{b}}$ & TNT3/OS3 $^{\mathrm{C}}$ & TNT4/OS4 $^{\mathrm{d}}$ \\
\hline LMS & $8.0 / 24.9$ & $5.6 / 17.3$ & $4.6 / 12.3$ & $4.4 / 9.2$ \\
UPS & $4.8 / 11.0$ & $3.5 / 7.9$ & $2.3 / 3.7$ & $3.5 / 6.2$ \\
DLPS & $4.4 / 11.8$ & $5.1 / 8.8$ & $2.4 / 6.0$ & $3.2 / 8.5$ \\
SS & $8.7 / 19.7$ & $5.7 / 11.7$ & $3.4 / 7.8$ & $2.3 / 6.0$ \\
MPNST & $4.1 / 12.5$ & $2.8 / 7.0$ & $3.6 / 8.0$ & $3.7 / 5.4$
\end{tabular}

${ }^{\mathrm{a}}$ Calculated from the date of first-line treatment onset

${ }^{\mathrm{b}}$ Calculated from the date of second-line treatment onset

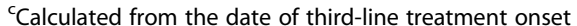

${ }^{\mathrm{d} C}$ Calculated from the date of fourth-line treatment onset

DLPS dedifferentiated liposarcomas, LMS leiomyosarcomas, MPNST malignant peripheral nerve sheath sarcomas, SS synovial sarcomas, UPS undifferentiated pleomorphic sarcomas
Table 4 Correlation between time to next treatment (TNT) and overall survival (OS)

\begin{tabular}{lcl}
\hline & Spearman's rho & $95 \% \mathrm{Cl}$ \\
\hline TNT1/OS1 $^{a}$ & 0.76 & $0.73-0.78$ \\
TNT2/OS2 $^{\text {b }}$ & 0.70 & $0.67-0.73$ \\
TNT3/OS3 $^{\text {c }}$ & 0.68 & $0.65-0.72$ \\
TNT4/OS4 $^{\text {d }}$ & 0.73 & $0.70-0.76$ \\
\hline
\end{tabular}

aCalculated from the date of first-line treatment onset

${ }^{\mathrm{b}}$ Calculated from the date of second-line treatment onset

'Calculated from the date of third-line treatment onset

${ }^{\mathrm{d}}$ Calculated from the date of fourth-line treatment onset

patients who received at least one systemic treatment (Table 6).

The following factors remained associated with an increased OS in the multivariate analysis: female sex, LMS, locoregional treatment of metastases, inclusion in a clinical trial, and administration of polychemotherapy in the first line of metastatic treatment (Table 6, Fig. 2). A grade 3 tumor at diagnosis remained associated with a decreased OS (Table 6, Fig. 2).

\section{Parameters correlated with 5-year survival}

To evaluate the parameters associated with a long survival, we excluded patients alive and with a follow-up inferior to 5 years, leading to the inclusion of 1619 patients in this analysis. A total of 224 patients were alive 5 years after the diagnosis of metastasis. The characteristics and patterns of this population are described in Tables 1 and 2, respectively.

The odds ratios and confidence intervals estimated by the logistic regression model for the factors significantly associated with the probability of 5 -year survival are presented in Fig. 3. The factors associated with a higher probability of 5-year survival were locoregional treatment of metastases $(\mathrm{OR}=7.41 ; 95 \% \mathrm{CI}, 4.42-12.41)$ and inclusion in a clinical trial $(\mathrm{OR}=1.59 ; 95 \% \mathrm{CI}, 1.04-$ 2.42). A grade 3 tumor at the time of diagnosis of metastasis was associated with a lower probability of 5 -year survival ( $\mathrm{OR}=0.32$; $95 \% \mathrm{CI}, 0.21-0.48)$.

To observe the impact of the locoregional treatment modality on the probability of 5-year survival, we replaced the binary variable "locoregional treatment: yes/ no" by a categorical variable detailing the type of locoregional treatment received (surgery, radiotherapy, radiofrequency, other, combination, or none). The following locoregional treatment modalities were particularly and significantly associated with a higher probability of 5year survival: surgery $(\mathrm{OR}=11.20 ; 95 \% \mathrm{CI}, 6.19-20.26)$, radiofrequency $(\mathrm{OR}=15.62 ; 95 \% \mathrm{CI}, 5.04-48.41)$, and combination of modalities (OR $=9.60$; 95\% CI, 5.3817.14). Other types of treatment, such as radiotherapy, were also correlated with a better probability of long survival; however, the effect was not significant. 
Table 5 Prognostic factors for time to next treatment

\begin{tabular}{|c|c|c|c|c|}
\hline \multirow[b]{2}{*}{ Covariate } & \multicolumn{2}{|c|}{$\underline{\text { Univariate analysis }}$} & \multicolumn{2}{|c|}{$\underline{\text { Multivariate analysis }}$} \\
\hline & $P$ & HR $(95 \% \mathrm{Cl})$ & $P$ & HR $(95 \% \mathrm{Cl})$ \\
\hline Sex (ref: Male) & 0.0014 & $0.835(0.747-0.933)$ & 0.0013 & $0.825(0.733-0.928)$ \\
\hline Age (ref: < 75 years old) & 0.0023 & $1.374(1.120-1.686)$ & - & - \\
\hline \multicolumn{5}{|l|}{ Histotype (ref: Other) } \\
\hline LMS & 0.5114 & $0.955(0.831-1.097)$ & - & - \\
\hline DLPS & 0.0068 & $1.357(1.088-1.692)$ & - & - \\
\hline MPNST & 0.3703 & $1.154(0.843-1.580)$ & - & - \\
\hline SS & 0.8580 & $0.983(0.811-1.191)$ & - & - \\
\hline UPS & 0.0375 & $1.243(1.013-1.525)$ & - & - \\
\hline Grade (ref: <3) & $<0.0001$ & $1.417(1.258-1.596)$ & $<0.0001$ & $1.372(1.218-1.546)$ \\
\hline Number of metastatic sites (ref: 1) & 0.1175 & $1.118(0.972-1.285)$ & - & - \\
\hline Liver metastasis (ref: no) & 0.1436 & $1.103(0.967-1.259)$ & - & - \\
\hline Locoregional treatment (ref: no) & $<0.0001$ & $0.496(0.442-0.556)$ & $<0.0001$ & $0.487(0.432-0.550)$ \\
\hline Clinical trial in first line (ref: no) & 0.6453 & $1.048(0.859-1.277)$ & - & - \\
\hline Anthracycline in first line (ref: no) & $<0.0001$ & $0.756(0.674-0.847)$ & - & - \\
\hline Polychemotherapy in first line (ref: no) & $<0.0001$ & $0.729(0.651-0.815)$ & $<0.0001$ & $0.743(0.660-0.836)$ \\
\hline
\end{tabular}

DLPS dedifferentiated liposarcomas, LMS leiomyosarcomas, MPNST malignant peripheral nerve sheath sarcomas, SS synovial sarcomas, UPS undifferentiated pleomorphic sarcomas

\section{Discussion}

The heterogeneity of STS has rarely been taken into account in the design of clinical trials to investigate systemic therapies in STS patients. Our results indicated that LMS clearly represented a distinct STS subgroup with a significantly better outcome in the advanced setting. Previous studies have shown worse outcomes for LMS than the results obtained in our current analysis. The largest study published to date was a retrospective analysis of 2185 patients with advanced STS treated in
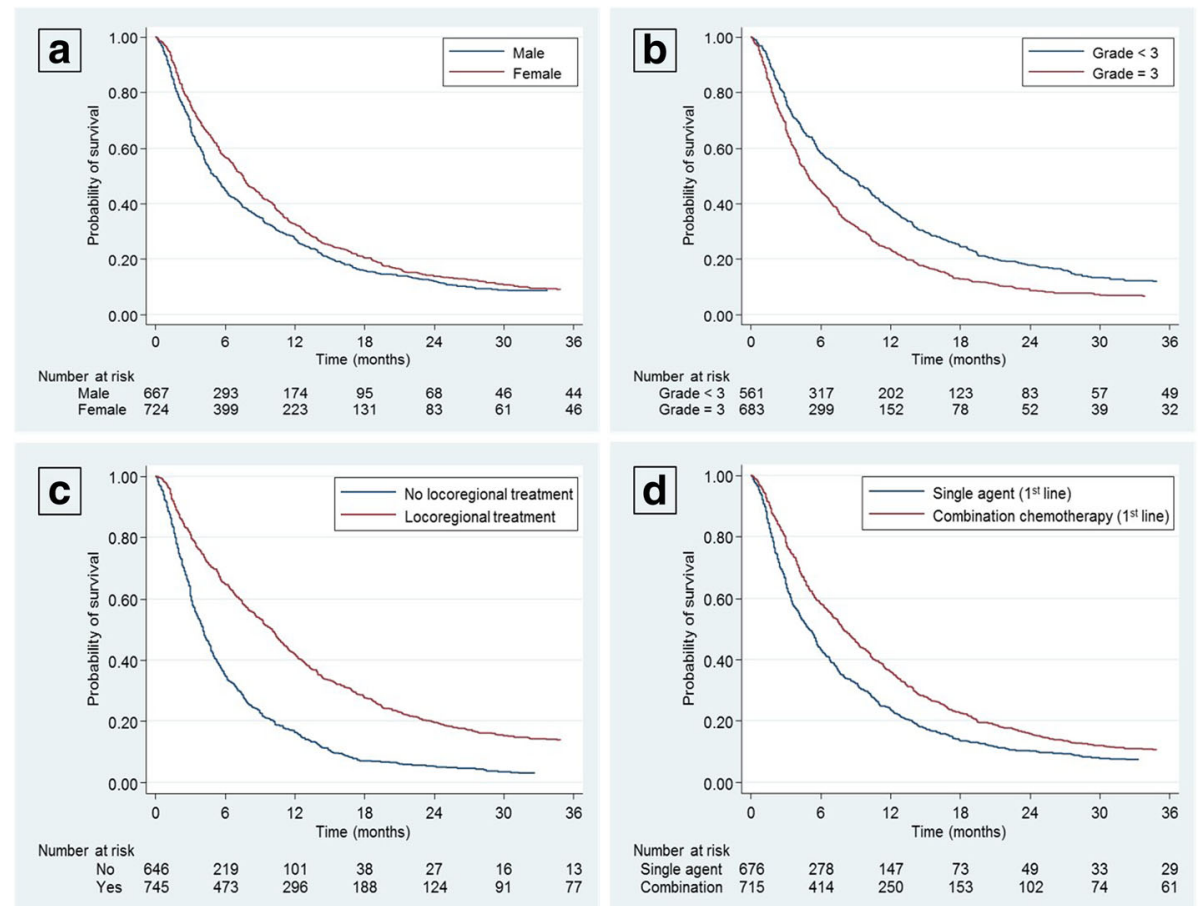

Fig. 1 Prognostic factors of time to next treatment - Kaplan-Meier curves. Kaplan-Meier Curves of time to next treatment according to (a) gender, (b) grade, (c) locoregional treatment of metastases, and (d) type of systemic treatment 
Table 6 Prognostic factors for overall survival

\begin{tabular}{|c|c|c|c|c|}
\hline \multirow[b]{2}{*}{ Covariate } & \multicolumn{2}{|c|}{ Univariate analysis } & \multicolumn{2}{|c|}{ Multivariate analysis } \\
\hline & $P$ & $\mathrm{HR}(95 \% \mathrm{Cl})$ & $P$ & HR $(95 \% \mathrm{Cl})$ \\
\hline Sex (ref: Male) & 0.0002 & $0.801(0.713-0.899)$ & 0.0003 & $0.792(0.698-0.900)$ \\
\hline Age (ref: < 75 years old) & 0.0024 & $1.389(1.123-1.717)$ & - & - \\
\hline \multicolumn{5}{|l|}{ Histotype (ref: Other) } \\
\hline LMS & 0.0004 & $0.765(0.659-0.888)$ & 0.0010 & $0.765(0.652-0.897)$ \\
\hline DLPS & 0.0269 & $1.291(1.030-1.619)$ & 0.2034 & $1.171(0.918-1.492)$ \\
\hline MPNST & 0.1368 & $1.273(0.926-1.751)$ & 0.2183 & $1.234(0.883-1.726)$ \\
\hline SS & 0.4738 & $1.074(0.883-1.307)$ & 0.0764 & $1.206(0.980-1.485)$ \\
\hline UPS & 0.0061 & $1.347(1.089-1.668)$ & 0.1839 & $1.168(0.929-1.469)$ \\
\hline Grade (ref: < 3) & $<0.0001$ & $1.692(1.491-1.920)$ & $<0.0001$ & $1.687(1.483-1.919)$ \\
\hline Number of metastatic sites (ref: 1) & 0.0136 & $1.200(1.038-1.387)$ & 0.0009 & $1.305(1.115-1.528)$ \\
\hline Liver metastasis (ref: no) & 0.1056 & $0.891(0.774-1.025)$ & - & - \\
\hline Locoregional treatment (ref: no) & $<0.0001$ & $0.412(0.365-0.465)$ & $<0.0001$ & $0.400(0.351-0.455)$ \\
\hline Clinical trial (ref: no) & $<0.0001$ & $0.750(0.653-0.862)$ & 0.0002 & $0.755(0.651-0.877)$ \\
\hline Off-label drugs (ref: no) & $<0.0001$ & $0.791(0.703-0.890)$ & - & - \\
\hline Anthracycline (ref: no) & 0.0046 & $0.838(0.741-0.947)$ & - & - \\
\hline Anthracycline in first line (ref: no) & 0.0127 & $0.861(0.765-0.968)$ & - & - \\
\hline Polychemotherapy in first line (ref: no) & 0.0003 & $0.804(0.715-0.902)$ & 0.0023 & $0.822(0.724-0.932)$ \\
\hline
\end{tabular}

DLPS dedifferentiated liposarcomas, LMS leiomyosarcomas, MPNST malignant peripheral nerve sheath sarcomas, SS synovial sarcomas, UPS undifferentiated pleomorphic sarcomas

the first-line studies of EORTC-STBSG; these patients showed no significant differences in terms of OS between LMS (492 cases) and the other histological subtypes, with a median OS of approximately 12 months [6]. However, this study, which focused only on first-line treatment, included patients diagnosed before the identification of the KIT mutation in gastrointestinal stromal tumors [4]. Therefore, a significant proportion of gastrointestinal stromal tumors, which are chemorefractory, were likely included in the LMS group. The better outcome of LMS may be explained by a specific biology but also by the potentially higher sensitivity to some anticancer agents such as gemcitabine, dacarbazine, or trabectedin. For instance, in a recent phase II randomized trial, patients with leiomyosarcomas of any origin benefited significantly from the combination of gemcitabine with dacarbazine, achieving a median progression-free survival (PFS) and OS of 4.9 and 13.8 months, respectively, versus 2.1 and 7.8 months, respectively, for the non-leiomyosarcoma subtypes [7]. Moreover, a large worldwide expanded access program for trabectedin showed a median OS of 16.2 months in 321 heavily pretreated leiomyosarcoma patients versus a median survival time of 11.9 months for the whole cohort of 903 patients [8].

We report here the first study assessing the outcomes of patients with advanced UPS. Some past reports included patients with malignant fibrous histiocytomas
(MFHs). However, a significant subset of tumors initially diagnosed as MFH showed a specific line of differentiation (lipogenic, neurogenic, myogenic, or nonsarcomatous) [9-12]. "MFH" is now considered an obsolete terminology and has been replaced by the term UPS, which is a diagnosis of exclusion. We found that patients with advanced UPS had the worst outcome with the shortest TNT and a median OS of only 11 months. These results illustrate the particular resistance to chemotherapy of this histological subset and an intrinsically more aggressive biology. Further investigations are needed to better understand the mechanisms of their tumorigenesis and to define more appropriate therapeutic strategies.

Approximately $45 \%$ of the 1575 patients who underwent systemic therapy received a combination chemotherapy regimen in the first-line setting. The first-line chemotherapy for advanced, metastatic, or nonresectable STS is typically based on single-agent doxorubicin [13]. Indeed, the majority of clinical studies comparing single agents with combinations failed to show an OS advantage but consistently showed improvement in the response rates and PFS [14, 15]. Interestingly, our analysis showed a significant impact of the use of combination chemotherapy on OS, with a hazard ratio of $0.822(0.724-0.932)$ and $P=0.0003$. Judson et al. [14] recently published the results of a randomized clinical trial evaluating doxorubicin as a single agent in the control 


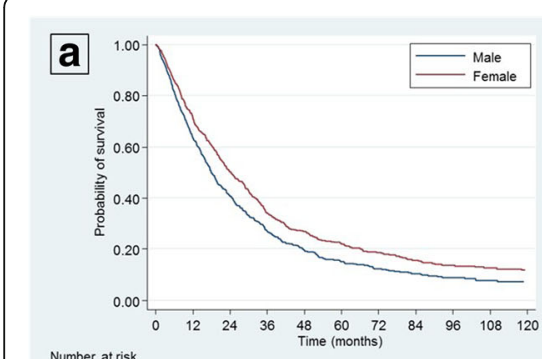

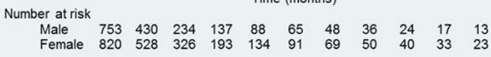
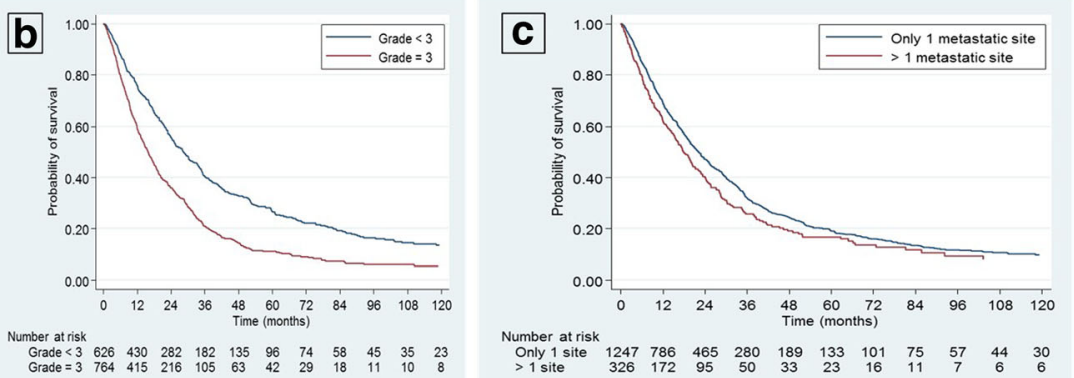

.
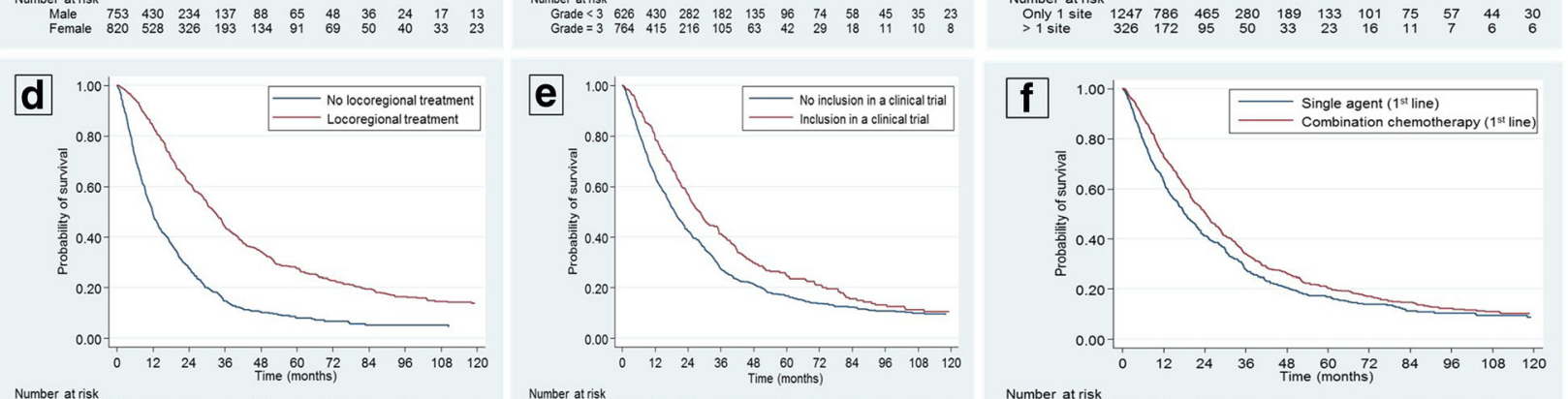

Number atrisk
No

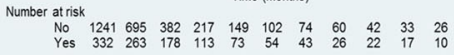

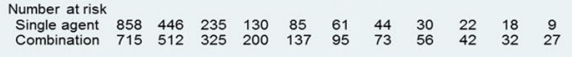

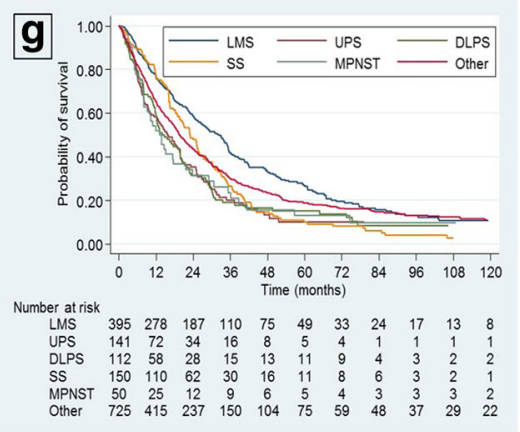

Fig. 2 Prognostic factors of overall survival - Kaplan-Meier curves. Kaplan-Meier curves of Overall survival according to (a) gender, (b) grade, (c) number of metastatic sites, (d) locoregional treatment of metastases, (e) inclusion in a clinical trial, (f) type of systemic treatment, (g) histological subtype

arm versus doxorubicin-ifosfamide in the experimental arm as a first-line treatment for advanced or metastatic STS. Although the Kaplan-Meier curves presented in the publication highlighted a difference between the two treatment arms in favor of polychemotherapy, the trial failed to detect a significant effect of polychemotherapy on OS, which was in contrast to our results. Our results suggest that the negative outcome of this study may simply be due to a lack of power as already suggested by Benjamin and Lee [16]. Indeed, by including 450 patients

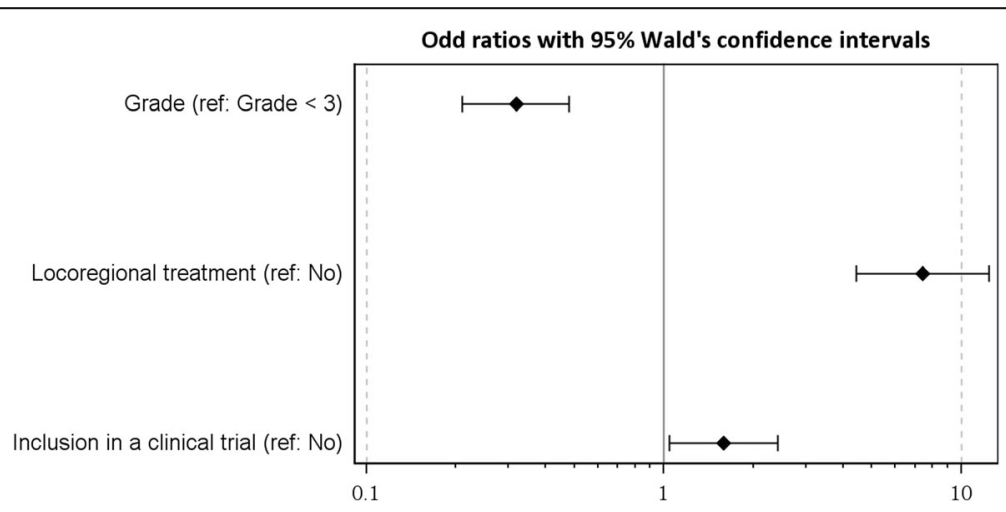

Fig. 3 Prognostic factors for 5-year survival - Odd ratios with 95\% Wald's confidence intervals 
and observing at least 366 events, the trial was designed to detect a maximum HR of 0.737. Due to the large size of our dataset, we were able to observe an HR of 0.822 . Based on their hypotheses, a total of 827 events would be required to detect a similar treatment effect in a randomized clinical trial. Although our study suggests a benefit in terms of OS, clinicians should also be aware that randomized trials have clearly demonstrated that combination chemotherapy is more toxic than singleagent doxorubicin with a potential significant impact on the quality of life $[14,15]$. Therefore, a combination of doxorubicin with a second drug such as ifosfamide should be used only after a careful discussion with the patient on the benefit/risk ratio of this approach, particularly when tumor shrinkage is expected to improve the symptoms or clinical benefits.

A high proportion of patients received more than two lines of systemic treatment. With the exception of leiomyosarcomas, our results indicate that the benefit of a greater than third-line regimen is very limited, with the median TNT and OS ranging between 2.3 and 3.7 months and 5.4 and 8.5 months, respectively. This result is consistent with the data from the PALETTE study, which led to the approval of pazopanib in advanced STS [17]. In that study, the number of previous lines of chemotherapy was a significant prognostic factor in the multivariate analysis for PFS with a significantly worse outcome in patients receiving pazopanib in the third- or fourth-line settings versus the first- or secondline settings. Given the potential toxicity and the moderate benefit of systemic therapy after failure of the second-line treatment, best supportive care should be considered as a reasonable option, particularly in patients with non-leiomyosarcoma histology and a poor performance status or patients who were not eligible to participate in a clinical trial. Notably, $50 \%$ of patients received an off-label drug during their treatment disease course. This result reflects the increasing evidence for the use of other drugs besides doxorubicin and ifosfamide in the sarcoma field. The most frequently prescribed off-label drug in this study was gemcitabine. Indeed, gemcitabine with or without docetaxel is commonly used in some specific sarcoma subsets, particularly in leiomyosarcomas and angiosarcomas [18-21], although neither of these drugs is approved for this indication. Another not yet approved drug that is frequently used in the sarcoma field is paclitaxel, which shows activity particularly in angiosarcomas $[22,23]$.

A significant proportion of patients with metastatic STS (27\%) did not receive any systemic therapy. An age $>75$ years was significantly associated with a lower probability of receiving any systemic treatment. Aging is associated with progressive functional declines, an increased prevalence of comorbidities, and a higher risk of cardiac and hematological toxicities related to anthracyclines [24-26]. These data may explain the reluctance of oncologists to use chemotherapy in elderly patients with STS and raises the question of the development of adapted chemotherapy regimens for elderly patients with advanced STS, such as low-dose cyclophosphamide [27] or liposomal doxorubicin [28].

A total of $49 \%$ of the patients received a loco-regional treatment of the metastasis, the most frequent of which were surgery followed by radiotherapy and radiofrequency ablation. The majority of these patients (71\%) had lung metastases. The published evidence on the role of locoregional treatments, such as pulmonary metastasectomy, is derived from a small number of studies with limited sample sizes [29]. Primary bone sarcomas, which may represent a distinct disease, are often included in these analyses. Our present study differed from previous publications because we used a larger database cohort, which increased the power of the multivariate analysis; additionally, we focused on STS exclusively to enhance the homogeneity of the study population. As suggested by previous studies, patients who underwent a locoregional metastasis treatment had improved survival in the multivariate analysis. Arguments have suggested that an observational study may not provide evidence that a difference in survival is attributable to the locoregional treatment and that only a randomized trial can answer the question. However, we observed that more than $80 \%$ of metastatic patients alive 5 years after the diagnosis of metastasis had received a locoregional treatment, versus $50 \%$ in the general population, and this parameter was most significantly associated with the probability of being alive at 5 years in the logistic regression model. Precisely, the descriptive analyses of the patients alive after 5 years suggest that surgery, radiofrequency, and a combination of different modalities are particularly beneficial in terms of survival. This hypothesis was confirmed by our sensibility analysis, since we found that the positive effect on the probability of 5-year survival was significant for these three treatment modalities only.

No data are available from randomized clinical trials to define how best to integrate the locoregional treatment of metastases in the management of patients with advanced disease. The most recent attempts were made by the European Organisation for Research and Treatment of Cancer (EORTC-Protocol 62933) with a randomized multicenter trial to assess metastasectomy alone versus induction chemotherapy followed by metastasectomy in a targeted sample size of 340 patients. Started in 1996, this trial was closed due to poor accrual in November of 2000. Notably, we report here the first large series of patients who received non-surgical locoregional treatment of metastases, including 254 patients treated with radiotherapy, 42 with radiofrequency ablation, and 320 with a 
combination of surgery plus radiotherapy or surgery plus radiofrequency ablation of metastases.

The gold standard endpoint in randomized clinical trials in oncology is OS. However, the use of a surrogate endpoint at an earlier stage in clinical trials would speed up the assessment of treatments and might reduce the cost of drug development. Studies that assess the use of alternative outcome measures, such as the response rate or PFS, as surrogate endpoints for OS in sarcoma patients showed only a modest if any correlation with PFS and OS [30,31]. This issue was recently illustrated with the pivotal trial that led to eribulin approval in patients with liposarcomas that showed a benefit in OS but not in PFS [32]. TNT is an established endpoint that is mostly applied in hematological malignancies and has recently been used in breast, colon, and prostate cancer [33-35]. The use of this parameter is predicated on the concept that a change in treatment usually occurs in response to a real change in the patient status by integrating the efficacy and toxicity components. In our study, we found a strong correlation between TNT and OS. The prospective validation of this endpoint as a surrogate for OS should be done in future studies.

\section{Conclusions}

This study reports the most comprehensive information related to the patterns of care and outcome of STS with advanced disease managed in the real-life setting. Limitations include its observational nature, which provides a lower level of evidence than a conventional clinical trial, the lack of data related to visceral sarcomas and GIST, and to the safety of therapeutic interventions. However, there are several lines of evidence indicating that observational studies usually do provide valid information and could be used to exploit well-designed databases [36].

\section{Additional file}

Additional file 1: Supplementary Methods. (DOCX $11 \mathrm{~kb}$ )

\section{Abbreviations}

DLPS: Dedifferentiated liposarcoma; LMS: Leiomyosarcoma; MFHs: Malignant fibrous histiocytomas; MPNST: Malignant peripheral nerve sheath tumors; OS: Overall survival; PFS: Progression-free survival; SS: Synovial sarcoma; STSs: Soft-tissue sarcomas; TNT: Time to next treatment; UPS: Undifferentiated pleomorphic sarcoma

\section{Acknowledgments}

The authors are grateful to Jean-Baptiste Courreges, Myriam Jean-Denis, and Nouria Mesli for their contribution to the management of the French Sarcoma Group database.

\section{Funding}

The present study has been funded by the French National Cancer Institute.

\section{Availability of data and materials}

The datasets supporting the conclusions of this article cannot be shared for confidentiality reasons. The METASARC database contains the most comprehensive information related to the outcome of STS with advanced disease and will be continuously updated to help clinicians identify the best therapeutic options for their patients. Queries related to factors such as the activity of a drug in a specific histological subtype can be sent by email (metasarc@bordeaux.unicancer.fr). Similarly, our results will also be available for investigators who need a reference for the response and outcome in the assessment of an investigational drug in a specific setting.

\section{Authors' contributions}

Study concepts and design: Al. Acquisition, analysis or interpretation of data: MS, ALC, JYB, IRC, OM, MT, SC, PT, DRV, PM, ES, CH, PS, MPS, CLP, AG, CB, FLL, Al. Drafting of the manuscript: MS, ALC, JYB, IRC, OM, MT, SC, PT, DRV, PM, $E S, C H, P S, M P S, C L P, A G, C B, F L L, A l$. Critical revision of the manuscript for important intellectual content: MS, ALC, JYB, IRC, OM, MT, SC, PT, DRV, PM, $E S, C H, P S, M P S, C L P, A G, C B, F L L, A l$. All authors have given final approval of the version to be published. All authors agree to be accountable for all aspects of the work in ensuring that questions related to the accuracy or integrity of any part of the work are appropriately investigated and resolved.

\section{Competing interests}

The authors declare that they have no competing interests.

\section{Consent for publication}

Not applicable.

Ethics approval and consent to participate

This study was approved by the ethics committee of the Comprehensive Cancer Center Institut Bergonié (Bordeaux, France).

\section{Publisher's Note}

Springer Nature remains neutral with regard to jurisdictional claims in published maps and institutional affiliations.

\section{Author details}

${ }^{1}$ Clinical and Epidemiological Research Unit, Institut Bergonié, Bordeaux, France. ${ }^{2}$ ISPED, INSERM U1219 Bordeaux Population Health Center, Epicene Team, Bordeaux, France. ${ }^{3}$ Department of Medicine, Institut Gustave Roussy, Villejui, France. ${ }^{4}$ Department of Medicine, Centre Leon Berard, Lyon, France. ${ }^{5}$ Department of Medicine, Institut Bergonié, Bordeaux, France. ${ }^{6}$ Department of Pathology, Institut Gustave Roussy, Villejuif, France. "Department of Pathology, Centre Leon Berard, Lyon, France. ${ }^{8}$ Department of Surgery, Centre Leon Berard, Lyon, France. ${ }^{9}$ Department of Surgery, Institut Bergonié, Bordeaux, France. ${ }^{10}$ Department of Surgery, Institut Gustave Roussy, Villejuif, France. ${ }^{11}$ Department of Radiotherapy, Institut Bergonié, Bordeaux, France.

${ }^{12}$ Department of Radiotherapy, Centre Leon Berard, Lyon, France.

${ }^{13}$ Department of Radiotherapy, Institut Gustave Roussy, Villejuif, France.

${ }^{14}$ Department of Pathology, Institut Bergonié, Bordeaux, France. ${ }^{15}$ Early Phase Trials and Sarcoma Units, Institut Bergonié, 229 Cours de l'Argonne, Bordeaux, France.

Received: 3 December 2016 Accepted: 3 March 2017

Published online: 10 April 2017

\section{References}

1. Coindre JM, Terrier P, Guillou L, et al. Predictive value of grade for metastasis development in the main histologic types of adult soft tissue sarcomas: a study of 1240 patients from the French Federation of Cancer Centers Sarcoma Group. Cancer. 2001;91:1914-26.

2. Fletcher C, Unni K, Mertens F, editors. World Health Organization Classification of Tumours Pathology and Genetics of Tumours of Soft Tissue and Bone. Lyon: IARC Press; 2013.

3. Trojani $\mathrm{M}$, Contesso $\mathrm{G}$, Coindre JM, et al. Soft-tissue sarcomas of adults; study of pathological prognostic variables and definition of a histopathological grading system. Int J Cancer. 1984;33:37-42.

4. Hirota S, Isozaki K, Moriyama Y, et al. Gain-of-function mutations of c-kit in human gastrointestinal stromal tumors. Science. 1998;279:577-80.

5. Schemper M, Kaider A, Wakounig S, Heinze G. Estimating the correlation of bivariate failure times under censoring. Statist Med. 2013;32:4781-90. 
6. Van Glabbeke M, van Oosterom AT, Oosterhuis JW, et al. Prognostic factors for the outcome of chemotherapy in advanced soft tissue sarcoma: an analysis of 2,185 patients treated with anthracycline-containing first-line regimens-a European Organization for Research and Treatment of Cancer Soft Tissue and Bone Sarcoma Group Study. J Clin Oncol. 1999;17:150-7.

7. García-Del-Muro X, López-Pousa A, Maurel J, et al. Randomized phase II study comparing gemcitabine plus dacarbazine versus dacarbazine alone in patients with previously treated soft tissue sarcoma: a Spanish Group for Research on Sarcomas study. J Clin Oncol. 2011;29:2528-33.

8. Samuels BL, Chawla S, Patel $\mathrm{S}$, et al. Clinical outcomes and safety with trabectedin therapy in patients with advanced soft tissue sarcomas following failure of prior chemotherapy: results of a worldwide expanded access program study. Ann Oncol. 2013;24:1703-9.

9. Coindre JM, Mariani O, Chibon F, et al. Most malignant fibrous histiocytomas developed in the retroperitoneum are dedifferentiated liposarcomas: a review of 25 cases initially diagnosed as malignant fibrous histiocytoma. Mod Pathol. 2003;16:256-62.

10. Fletcher CD. Pleomorphic malignant fibrous histiocytoma: fact or fiction? A critical reappraisal based on 159 tumors diagnosed as pleomorphic sarcoma. Am J Surg Pathol. 1992;16:213-28.

11. Fletcher CD, Gustafson P, Rydholm A, Willén H, Akerman M. Clinicopathologic re-evaluation of 100 malignant fibrous histiocytomas: prognostic relevance of subclassification. J Clin Oncol. 2001;19:3045-50.

12. Oda Y, Tamiya S, Oshiro Y, et al. Reassessment and clinicopathological prognostic factors of malignant fibrous histiocytoma of soft parts. Pathol Int. 2002;52:595-606.

13. ESMO/European Sarcoma Network Working Group. Soft tissue and visceral sarcomas: ESMO Clinical Practice Guidelines for diagnosis, treatment and follow-up. Ann Oncol. 2014;25 Suppl 3:iii102-12.

14. Judson I, Verweij J, Gelderblom H, et al. Doxorubicin alone versus intensified doxorubicin plus ifosfamide for first-line treatment of advanced or metastatic soft-tissue sarcoma: a randomised controlled phase 3 trial. Lancet Oncol. 2014;15:415-23.

15. Santoro A, Tursz T, Mouridsen $H$, et al. Doxorubicin versus CYVADIC versus doxorubicin plus ifosfamide in first-line treatment of advanced soft tissue sarcomas: a randomized study of the European Organization for Research and Treatment of Cancer Soft Tissue and Bone Sarcoma Group. J Clin Oncol. 1995;13:1537-45.

16. Benjamin RS, Lee JJ. One step forward, two steps back. Lancet Oncol. 2014; 15:366-7.

17. van der Graaf WT, Blay JY, Chawla SP, et al. Pazopanib for metastatic softtissue sarcoma (PALETTE): a randomised, double-blind, placebo-controlled phase 3 trial. Lancet. 2012;379:1879-86.

18. Ducoulombier A, Cousin S, Kotecki N, Penel N. Gemcitabine-based chemotherapy in sarcomas: a systematic review of published trials. Crit Rev Oncol Hematol. 2016;98:73-80.

19. Stacchiotti S, Palassini E, Sanfilippo R, et al. Gemcitabine in advanced angiosarcoma: a retrospective case series analysis from the Italian Rare Cancer Network. Ann Oncol. 2012;23:501-8.

20. von Mehren M, Randall RL, Benjamin RS, et al. Soft Tissue Sarcoma, Version 2.2016, NCCN Clinical Practice Guidelines in Oncology. J Natl Compr Canc Netw. 2016;14:758-86.

21. Duffaud F, Pautier $P$, Bui $B$, et al. A pooled analysis of the final results of the two randomized phase II studies comparing gemcitabine $(G)$ vs. gemcitabine + docetaxel $(G+D)$ in patients (pts) with metastatic/relapsed leiomyosarcoma (LMS). Ann Oncol. 2010;21 Suppl 8:viii408-16. doi:10.1093/ annonc/mdq536.

22. Italiano A, Cioffi A, Penel N, et al. Comparison of doxorubicin and weekly paclitaxel efficacy in metastatic angiosarcomas. Cancer. 2012;118:3330-6.

23. Penel N, Bui BN, Bay JO, et al. Phase II trial of weekly paclitaxel for unresectable angiosarcoma: the ANGIOTAX Study. J Clin Oncol. 2008;26: 5269-74.

24. Swain SM, Whaley FS, Ewer MS. Congestive heart failure in patients treated with doxorubicin: a retrospective analysis of three trials. Cancer. 2003;97: 2869-79.

25. Fumoleau $P$, Roché $H$, Kerbrat $P$, et al. Long-term cardiac toxicity after adjuvant epirubicin-based chemotherapy in early breast cancer: French Adjuvant Study Group results. Ann Oncol. 2006;17:85-92

26. Shayne M, Culakova E, Poniewierski MS, et al. Dose intensity and hematologic toxicity in older cancer patients receiving systemic chemotherapy. Cancer. 2007;110:1611-20.
27. Mir O, Domont J, Cioffi A, et al. Feasibility of metronomic oral cyclophosphamide plus prednisolone in elderly patients with inoperable or metastatic soft tissue sarcoma. Eur J Cancer. 2011;47:515-9.

28. Judson I, Radford JA, Harris M, et al. Randomised phase II trial of pegylated liposomal doxorubicin (DOXIL/CAELYX) versus doxorubicin in the treatment of advanced or metastatic soft tissue sarcoma: a study by the EORTC Soft Tissue and Bone Sarcoma Group. Eur J Cancer. 2001;37:870-7.

29. Treasure T, Fiorentino F, Scarci M, Møller H, Utley M. Pulmonary metastasectomy for sarcoma: a systematic review of reported outcomes in the context of Thames Cancer Registry data. BMJ Open. 2012;2: e001736.

30. Savina $M$, Litière $S$, Penel $N$, et al. Surrogate properties of survival endpoints in metastatic soft-tissue sarcoma: a meta-analysis. J Clin Oncol. 2015; 33(Suppl):abstr: 10547.

31. Zer A, Prince RM, Amir E, Abdul RA. Evolution of randomized trials in advanced/metastatic soft tissue sarcoma: end point selection, surrogacy, and quality of reporting. J Clin Oncol. 2016;34:1469-75.

32. Schöffski P, Chawla S, Maki RG, et al. Eribulin versus dacarbazine in previously treated patients with advanced liposarcoma or leiomyosarcoma: a randomised, open-label, multicentre, phase 3 trial. Lancet. 2016;387:1629-37.

33. Chudley L, McCann K, Mander A, et al. DNA fusion-gene vaccination in patients with prostate cancer induces high-frequency CD8(+) T-cell responses and increases PSA doubling time. Cancer Immunol Immunother. 2012;61:2161-70.

34. Liang C, Li L, Fraser CD, et al. The treatment patterns, efficacy, and safety of nab $\left.{ }^{(}\right)$-paclitaxel for the treatment of metastatic breast cancer in the United States: results from health insurance claims analysis. BMC Cancer. 2015;15:1019.

35. Teng $\mathrm{CL}$, Wang $\mathrm{CY}$, Chen $\mathrm{YH}$, Lin $\mathrm{CH}$, Hwang WL. Optimal sequence of irinotecan and oxaliplatin-based regimens in metastatic colorectal cancer: a population-based observational study. PLoS One. 2015;10:e0135673.

36. Benson K, Hartz AJ. A comparison of observational studies and randomized, controlled trials. N Engl J Med. 2000;342:1878-86.

\section{Submit your next manuscript to BioMed Central and we will help you at every step:}

- We accept pre-submission inquiries

- Our selector tool helps you to find the most relevant journal

- We provide round the clock customer support

- Convenient online submission

- Thorough peer review

- Inclusion in PubMed and all major indexing services

- Maximum visibility for your research

Submit your manuscript at www.biomedcentral.com/submit
) Biomed Central 